\title{
The double edge of cancer immunotherapy
}

\author{
Immune-related adverse effects are understudied and not easily treatable risks of cancer immunotherapy. Concerted \\ research efforts to understand the mechanisms of immunotherapy-triggered responses are crucial for developing \\ better treatments.
}

$\mathrm{m}$ mmunotherapies have shown unprecedented efficacy in the treatment of several cancers, including melanoma and lymphoma, and they are now in clinical trials for many others. This type of treatment can differ in form, ranging from antibodies to vaccines and engineered lymphocytes. It also varies in its targets, dosages and how it is combined with other therapies. Despite their diversity, immunotherapies share a common mechanism of action involving the stimulation of immune responses. Not surprisingly, they also share a common complication: immune-related adverse effects.

For ipilimumab, these complications include gastrointestinal, hepatic and skin inflammation ranging from mild to lethal. Various methods have been developed to help physicians to recognize and treat these side effects. Approaches include education of clinicians and patients, guidelines for monitoring treatment and protocols to consult with experts on unusual complications. Despite these measures, clinical options for managing immune-related complications remain limited. They are currently treated with steroids and immunosuppressants, just as classical autoimmune and inflammatory diseases are, but with less success.

One challenge of predicting and diagnosing immunotherapy-induced adverse immune effects is that they can differ considerably in terms of their time of onset, severity, duration and which tissues they affect. Managing these reactions would be easier if the underlying causes were better understood. Researchers are similarly exploring determinants of the positive anti-tumor immune response. Some determinants of a positive response may overlap with those that produce an adverse response, but it's important to understand how the two phenomena differ.

Many researchers have sought to find biomarkers that predict who will respond positively to cancer immunotherapy, and a number of biomarkers have already been reported (J. Immunother. Cancer 4, 3, 2016). Biomarkers might similarly predict immune-related adverse reactions, but the hunt for these markers has had less momentum and has yielded fewer candidates. One rare exception is the recent link found between microbiome bacteria belonging to the Bacteroidetes phylum and resistance to the development of checkpoint-blockade-induced colitis (Nat. Commun. 7, 10391, 2016).

Patients with a past history of autoimmune and inflammatory conditions are often excluded from immunotherapy clinical trials because they are assumed to be at high risk of adverse immune effects. But it's worth noting that in a study of ipilimumab, only a fraction of such patients experienced exacerbation of immune toxicity with this drug, and this unwanted outcome was largely manageable with standard care. However, the rate of positive response in this subgroup was as good as that among patients with melanoma involved in other trials (JAMA Oncol. 2, 234-
$240,2016)$. Thus, there is hope that the good and bad immune reactions unleashed by immunotherapies can be controlled separately. More support for this idea comes from data on allogeneic bone marrow transplants, used to treat hematological malignancies, in which the goal is to induce graft-versus-leukemia response while avoiding graft-versus-host disease. The optimization of such transplants over decades has greatly diminished adverse reactions in acute myeloid leukemia and plasma cell disorders (Tissue Antigens 81, 183-193, 2013).

More research into the mechanism of immune reactions induced by immunotherapy is needed, but relatively little is being done. William Murphy, a dermatology professor at the University of California, Davis, who has sat on eight government grant-application review committees focused on immunotherapy, said in a New York Times article published on 3 December 2016 that only 3 out of 500 immunotherapy research proposals that he reviewed focused on the possible toxic side effects of this type of treatment.

Although mouse models can be instrumental in dissecting mechanisms and testing treatments of human disease, they rarely recapitulate the adverse immune responses seen in patients. Better animal models are needed. Ultimately, a lot can be learned about side effects from the clinic. But researchers first need to decipher at which time points to assess adverse reactions to immunotherapy, which types of biopsy contain the most relevant information and what confounding factors to watch out for. Collaborations between clinical, academic, regulatory, funding and biopharma organizations are necessary to collect, share and analyze data in a coordinated way. Protocols exist for monitoring and reporting adverse effects in clinical trials, and for approved treatments that require postmarketing surveillance owing to their high risk-to-benefit ratio. But the primary goal of these protocols is to evaluate, treat and mitigate risks using conventional approaches-not to develop more ways to eliminate them. A multi-center research network with a database of clinical and research data would provide improved statistical power for analyses. There are already several organizations that could help foster this kind of network, such as the Cancer Immunotherapy Trials Network and Parker Institute for Cancer Immunotherapy.

Cancer is a complex biological process. It takes an equally versatile biological process to fight it: the immune system. Decades of research have generated dynamic tools for selecting optimal chemotherapy options, managing side effects and predicting outcomes. This is not yet the case for immunotherapies. We still have much to learn about the complex physiological chain reactions that immunotherapy triggers. Those involved in the field can move it forward by raising awareness of the problem of adverse events and sharing data, ideas and resources. 